\title{
ON THE LENGTH SPECTRUM TEICHMÜLLER SPACES OF RIEMANN SURFACES OF INFINITE TYPE
}

\begin{abstract}
ERINA KINJO
Abstract. On the Teichmüller space $T\left(R_{0}\right)$ of a hyperbolic Riemann surface $R_{0}$, we consider the length spectrum metric $d_{L}$, which measures the difference of hyperbolic structures of Riemann surfaces. It is known that if $R_{0}$ is of finite type, then $d_{L}$ defines the same topology as that of Teichmüller metric $d_{T}$ on $T\left(R_{0}\right)$. In 2003, H. Shiga extended the discussion to the Teichmüller spaces of Riemann surfaces of infinite type and proved that the two metrics define the same topology on $T\left(R_{0}\right)$ if $R_{0}$ satisfies some geometric condition. After that, Alessandrini-Liu-Papadopoulos-Su proved that for the Riemann surface satisfying Shiga's condition, the identity map between the two metric spaces is locally bi-Lipschitz.

In this paper, we extend their results; that is, we show that if $R_{0}$ has bounded geometry, then the identity map $\left(T\left(R_{0}\right), d_{L}\right) \rightarrow\left(T\left(R_{0}\right), d_{T}\right)$ is locally bi-Lipschitz.
\end{abstract}

\section{INTRODUCTION}

We say that a Riemann surface is hyperbolic if its universal cover is the Poincaré disk. For a hyperbolic Riemann surface $R_{0}$, consider a pair $(R, f)$ of a Riemann surface $R$ and a quasiconformal mapping $f$ from $R_{0}$ to $R$. Such pairs $\left(R_{1}, f_{1}\right)$ and $\left(R_{2}, f_{2}\right)$ are called Teichmüller equivalent if there exists a conformal mapping $h: R_{1} \rightarrow R_{2}$ that is homotopic to $f_{2} \circ f_{1}^{-1}$, where the homotopy does not necessarily fix points of the ideal boundary $\partial R_{0}$. The Teichmüller space $T\left(R_{0}\right)$ of $R_{0}$ is the set of all Teichmüller equivalence classes.

$T\left(R_{0}\right)$ has a complete metric $d_{T}$ called the Teichmüller metric. It is defined by

$$
d_{T}\left(\left[R_{1}, f_{1}\right],\left[R_{2}, f_{2}\right]\right)=\log \inf _{f} K(f),
$$

where the infimum is taken over all quasiconformal mappings from $R_{1}$ to $R_{2}$ that are homotopic to $f_{2} \circ f_{1}^{-1}$, and $K(f)$ is the maximal dilatation of $f$. This means that $d_{T}$ measures the difference of complex structures of Riemann surfaces in $T\left(R_{0}\right)$.

We introduce another metric on $T\left(R_{0}\right)$. Let $\mathcal{C}\left(R_{0}\right)$ be the set of non-trivial and non-peripheral closed curves in $R_{0}$. We define the length spectrum metric $d_{L}$ by

$$
d_{L}\left(\left[R_{1}, f_{1}\right],\left[R_{2}, f_{2}\right]\right)=\log \sup _{\alpha \in \mathcal{C}\left(R_{0}\right)} \max \left\{\frac{\ell_{R_{1}}\left(\left[f_{1}(\alpha)\right]\right)}{\ell_{R_{2}}\left(\left[f_{2}(\alpha)\right]\right)}, \frac{\ell_{R_{2}}\left(\left[f_{2}(\alpha)\right]\right)}{\ell_{R_{1}}\left(\left[f_{1}(\alpha)\right]\right)}\right\},
$$

where $\left[f_{i}(\alpha)\right]$ is a geodesic which is freely homotopic to $f_{i}(\alpha)$, and $\ell_{R_{i}}(\cdot)$ is the hyperbolic length $(i=1,2)$. By definition, $d_{L}\left(\left[R_{1}, f_{1}\right],\left[R_{2}, f_{2}\right]\right)=0$ if and only if

Received by the editors September 23, 2016, and, in revised form, October 2, 2017.

2010 Mathematics Subject Classification. Primary 30F60; Secondary 32G15.

Key words and phrases. Length spectrum, Teichmüller metric, Riemann surface of infinite type. 
$\ell_{R_{1}}\left(\left[f_{1}(\alpha)\right]\right)=\ell_{R_{2}}\left(\left[f_{2}(\alpha)\right]\right)$ for any $\alpha \in \mathcal{C}\left(R_{0}\right)$. Hence $d_{L}$ measures the difference of hyperbolic structures of Riemann surfaces in $T\left(R_{0}\right)$.

Remark 1.1. Let $\mathcal{S}\left(R_{0}\right)$ be the set of non-trivial and non-peripheral simple closed curves in $R_{0}$. Thurston [16] showed that

$$
d_{L}\left(\left[R_{1}, f_{1}\right],\left[R_{2}, f_{2}\right]\right)=\log \sup _{\alpha \in \mathcal{S}\left(R_{0}\right)} \max \left\{\frac{\ell_{R_{1}}\left(\left[f_{1}(\alpha)\right]\right)}{\ell_{R_{2}}\left(\left[f_{2}(\alpha)\right]\right)}, \frac{\ell_{R_{2}}\left(\left[f_{2}(\alpha)\right]\right)}{\ell_{R_{1}}\left(\left[f_{1}(\alpha)\right]\right)}\right\} .
$$

In 1972, Sorvali [15] defined the metric $d_{L}$ and showed the following.

Lemma 1.2 ([15]). For any $\left[R_{1}, f_{1}\right],\left[R_{2}, f_{2}\right] \in T\left(R_{0}\right)$,

$$
d_{L}\left(\left[R_{1}, f_{1}\right],\left[R_{2}, f_{2}\right]\right) \leq d_{T}\left(\left[R_{1}, f_{1}\right],\left[R_{2}, f_{2}\right]\right)
$$

holds.

In 1986, Li [10] proved that $d_{T}$ and $d_{L}$ define the same topology on $T\left(R_{0}\right)$ if $R_{0}$ is a compact Riemann surface, and in 1999, Liu [11] showed that the same holds for any Riemann surface of finite type, i.e., compact surface from which at most finitely many points have been removed. In 2003, Shiga 14 showed that there exists a Riemann surface $R_{0}$ of infinite type such that $d_{T}$ and $d_{L}$ define different topologies on $T\left(R_{0}\right)$. After that, Liu-Sun-Wei [13, Kinjo [8], and Evren [6] gave new examples of Teichmüller spaces on which the two metrics define different topologies. Each Riemann surface they considered has a sequence of points whose injective radii either diverge or vanish. Here, the injective radius $\operatorname{inj}_{r}(x)$ of $x \in R_{0}$ is defined as follows:

$$
\operatorname{inj}_{r}(x)=\sup \{r>0 \mid \text { The } r \text {-neighborhood of } x \text { is homeomorphic to a disk. }\}
$$

First, we describe when $d_{T}$ and $d_{L}$ define the same topology on the Teichmüller space. In 2003, Shiga gave a sufficient condition (14]) for the two metrics to define the same topology. (We call the assumption of the following theorem Shiga's condition.)

Theorem 1.3 ([14, Theorem 1.2). Let $R_{0}$ be a Riemann surface. Assume that there exists a pants decomposition $R_{0}=\bigcup_{k=1}^{\infty} P_{k}$ satisfying the following conditions.

(1) Each connected component of $\partial P_{k}(k=1,2,3 \ldots)$ is either a puncture or a simple closed geodesic of $R_{0}$.

(2) There exists a constant $M>0$ such that if $\alpha$ is a boundary geodesic of some $P_{k}$, then

$$
0<M^{-1}<\ell_{R_{0}}(\alpha)<M
$$

holds.

Then $d_{T}$ and $d_{L}$ define the same topology on $T\left(R_{0}\right)$.

In our previous paper $(9,2014)$, we gave a new sufficient condition. Before mentioning it, we shall define bounded geometry of Riemann surfaces. First, we define a cusp neighborhood in $R_{0}$ as a punctured disc bounded by a horocycle such that its area is one and the length of horocycle is one. Let $R_{0}^{\prime}$ be a subsurface of $R_{0}$ obtained by removing all cusp neighborhoods from $R_{0}$.

Definition 1.4. A Riemann surface $R_{0}$ has bounded geometry if there exists a constant $M>0$ satisfying the condition (BG): any (non-trivial and non-peripheral) closed geodesic has length greater than $1 / M$ and every point $x$ in $R_{0}^{\prime}$ has a closed curve based on $x$ with the length less than $M$. 
Note that a Riemann surface $R_{0}$ has bounded geometry if and only if there exists a constant $M>0$ such that for any point $x \in R_{0}^{\prime}, 1 / M<\operatorname{inj}_{r}(x)<M$ holds.

Now the new condition in our previous paper is the following:

Theorem 1.5 (9], Corollary 1.5). Let $R_{0}$ be a Riemann surface with bounded geometry. Suppose $R_{0}$ has finite genus. Then $d_{T}$ and $d_{L}$ defines the same topology on $T\left(R_{0}\right)$.

Since the Riemann surface of Theorem 1.5 is of finite genus, it does not extend Shiga's Theorem. However, 1.5 does not restrict his condition. There exist Riemann surfaces satisfying our condition but not satisfying his condition. See Section 2 of [8] or Section 2 of 9 .

Next we consider Lipschitz continuity between the two metric spaces. AlessandriniLiu-Papadopoulos-Su ([2]) proved the following.

Theorem 1.6 ([2], Theorem 1.4). Let $R_{0}$ be a Riemann surface satisfying Shiga's condition. Then the identity map $\left(T\left(R_{0}\right), d_{L}\right) \rightarrow\left(T\left(R_{0}\right), d_{T}\right)$ is locally bi-Lipschitz.

In this paper, we extend the above three theorems 1.3, 1.5, and 1.6. Our main result is the following.

Theorem 1.7. Let $R_{0}$ be a Riemann surface with bounded geometry. Then the identity map $\left(T\left(R_{0}\right), d_{L}\right) \rightarrow\left(T\left(R_{0}\right), d_{T}\right)$ is locally bi-Lipschitz.

To make it easier to read this paper, we describe the outline of the proof of Theorem 1.7 For a Riemann surface $R_{0}$ with bounded geometry, let $M>0$ be a constant satisfying the condition (BG) (in Definition 1.4). We would like to see that $\frac{1}{C} d_{T}(p, q) \leq d_{L}(p, q) \leq C d_{T}(p, q)$ for any $p, q \in B\left(p_{0}, r\right)$, where $p_{0}=\left[R_{0}, i d\right]$ is a basepoint of $T\left(R_{0}\right), B\left(p_{0}, r\right)=\left\{p \in R_{0} \mid d_{L}\left(p, p_{0}\right)<r\right\}$ is some neighborhood (with respect to $\left.d_{L}\right)$ of $p_{0}$ and $C=C(M, r) \geq 1$. By Sorvali's Lemma, $d_{L}(p, q) \leq d_{T}(p, q)$ holds for any $p, q \in T\left(R_{0}\right)$, thus it is sufficient to show that $\frac{1}{C} d_{T}(p, q) \leq d_{L}(p, q)$. In proving it, the following is a Key Lemma.

Lemma 1.8. Let $R_{0}$ be a Riemann surface with bounded geometry. Then $R_{0}$ is decomposed into pairs of pants $\mathcal{P}=\left\{P_{n}\right\}_{n=1}^{\infty}$, (where some component of $\partial P_{n}$ may be a puncture) and hyperbolic right hexagons $\mathcal{H}=\left\{H_{m}\right\}_{m=1}^{\infty}$ satisfying the following conditions:

(1) $\stackrel{\circ}{P}_{n} \cap \stackrel{\circ}{P}_{k}=\emptyset(n \neq k), \stackrel{\circ}{P}_{n} \cap \stackrel{\circ}{H}_{m}=\emptyset, \stackrel{\circ}{H}_{m} \cap \stackrel{\circ}{H}_{l}=\emptyset(m \neq l)$ and $R_{0}=\bigcup_{n=1}^{\infty} P_{n} \cup \bigcup_{m=1}^{\infty} H_{m}$. Here, every other edge of $H_{m}(m=1,2, \ldots)$ is contained in some $\partial P_{n}$.

(2) The lengths of all closed geodesics in $\left\{\partial P_{n}\right\}_{n=1}^{\infty}$ are uniformly bounded from above and below.

(3) The lengths of all edges of hexagons $\left\{H_{m}\right\}_{m=1}^{\infty}$ are uniformly bounded from above and below.

If $R_{0}$ satisfies Shiga's condition, then $R_{0}$ can be decomposed by the above pairs of pants and hexagons. However, the converse is not true; that is, even if $R_{0}$ can be decomposed by those, it does not necessarily satisfy Shiga's condition (cf. [8], $\S 2)$.

We prove this lemma in Section 2, And we prove Theorem 1.7 in Section 3 by taking the following steps.

Step 1. Take two arbitrary points $p_{1}=\left[R_{1}, f_{1}\right], p_{2}=\left[R_{2}, f_{2}\right] \in B\left(p_{0}, r\right)$. Decompose $R_{1}$ into pairs of pants $\mathcal{P}_{1}$ and right hexagons $\mathcal{H}_{1}$ by Lemma 1.8. Also, 
decompose $R_{2}$ into pairs of pants $\mathcal{P}_{2}$ corresponding to $\mathcal{P}_{1}$ and right hexagons $\mathcal{H}_{2}$ corresponding to $\mathcal{H}_{1}$.

Step 2. Construct a quasiconformal mapping $\varphi: \mathcal{H}_{1} \rightarrow \mathcal{H}_{2}$ such that $K(\varphi)<$ $C(M, r)$, where $C(M, r) \rightarrow 1$ as $r \rightarrow 0$. We use Bishop's lemmas.

Step 3. Construct a quasiconformal mapping $\varphi: \mathcal{P}_{1} \rightarrow \mathcal{P}_{2}$ such that $K(\varphi)<$ $C(M, r)$, where $C(M, r) \rightarrow 1$ as $r \rightarrow 0$. In constructing, take notice of the twist along any closed geodesic $\alpha$ of $\partial P$ for any $P \in \mathcal{P}_{1}$. We use the fact that the twist is uniformly bounded since $d_{L}\left(p_{1}, p_{2}\right)<2 r$ and $\ell_{R_{1}}(\alpha)$ is uniformly bounded.

In these steps, we obtain a quasiconformal mapping $\varphi: R_{1} \rightarrow R_{2}$ such that $K(\varphi)<C(M, r)$, where $C(M, r) \rightarrow 1$ as $r \rightarrow 0$. We can check that $\varphi$ is homotopic to $f_{2} \circ f_{1}^{-1}$. This means $d_{T}\left(p_{1}, p_{2}\right)<\log C(M, r)$, where $\log C(M, r) \rightarrow 0$ as $r \rightarrow 0$, thus $d_{T}\left(p_{1}, p_{2}\right) \leq C d_{L}\left(p_{1}, p_{2}\right)$.

\section{Proof of the Key Lemma}

In this section, we prove Lemma 1.8. First, we take disjoint simple closed geodesics all over $R_{0}$. Here, $R_{0}^{\prime}$ is a subsurface of $R_{0}$ obtained by removing all cusp neighborhoods from $R_{0}$.

Lemma 2.1. Let $R_{0}$ be a Riemann surface with bounded geometry and let $M>0$ be a constant satisfying condition (BG) in Definition 1.4. Then there exists a family of pairwise disjoint and simple closed geodesics $\mathcal{G}:=\left\{\alpha_{n}\right\}_{n=0}^{\infty}$ in $R_{0}$ satisfying following conditions.

(1) $1 / M<\ell_{R_{0}}\left(\alpha_{n}\right)<M$ for any $\alpha_{n} \in \mathcal{G}$.

(2) There exists a constant $D=D(M)>0$ such that for any $x \in R_{0}^{\prime}$, $d_{R_{0}}\left(x, \alpha_{n}\right)<D$ holds for some $\alpha_{n} \in \mathcal{G}$, where $d_{R_{0}}($,$) is hyperbolic dis-$ tance on $R_{0}$.

Proof. By the definition of bounded geometry, for any $x \in R_{0}^{\prime}$, there exists a simple closed curve $c_{x}$ based on $x$ with $1 / M<\ell_{R_{0}}\left(c_{x}\right)<M$. First, we take some constant $D=D(M)>0$ as follows. For each $x \in R_{0}^{\prime}$, put

$$
d_{x}=\max _{c_{x}} d_{R_{0}}\left(\left[c_{x}\right], x\right),
$$

where $c_{x}$ is taken over all simple closed curves based on $x$ with the length $\epsilon$ $(1 / M, M)$, and $\left[c_{x}\right]$ is a closed geodesic homotopic to $c_{x}$.

Claim 2.2. $d_{x}<2 \log (\sqrt{2} M)+\frac{1}{2} M$ holds for any $x \in R_{0}^{\prime}$.

Proof. For an arbitrary point $x \in R_{0}^{\prime}$, take an arbitrary simple closed curve $c_{x}$ based on $x$ with length $\in(1 / M, M)$. If $\left[c_{x}\right] \cap c_{x} \neq \emptyset$, then $d_{R_{0}}\left(\left[c_{x}\right], x\right)<\frac{1}{2} \ell_{R_{0}}\left(c_{x}\right)<\frac{1}{2} M$. If $\left[c_{x}\right] \cap c_{x}=\emptyset$, then there exists a cylinder $C_{x}$ bounded by $\left[c_{x}\right]$ and $c_{x}$. Let $r_{x}>0$ be the distance from $\left[c_{x}\right]$ to $c_{x}$. In the cylinder $C_{x}$, take an $r_{x}$-neighborhood of $\left[c_{x}\right]$ (Figure 1), i.e.,

$$
C_{x}^{\prime}=\left\{y \in C_{x} \mid d_{R_{0}}\left(\left[c_{x}\right], y\right)<r_{x}\right\}
$$

Put $\zeta:=\partial C_{x}^{\prime}-\left[c_{x}\right]$, then

$$
\ell_{R_{0}}(\zeta)=\ell_{R_{0}}\left(\left[c_{x}\right]\right) \cdot \cosh r_{x}
$$




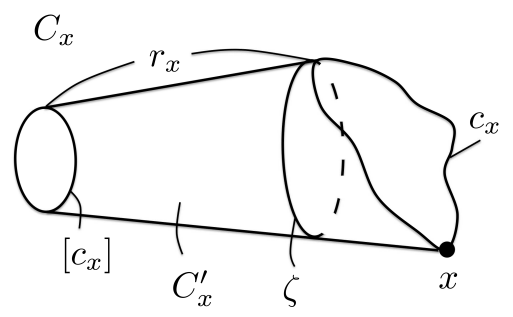

Figure 1 . A cylinder $C_{x}$ bounded by $\left[c_{x}\right]$ and $c_{x}$, and a subcylin$\operatorname{der} C_{x}^{\prime} \subset C_{x}$.

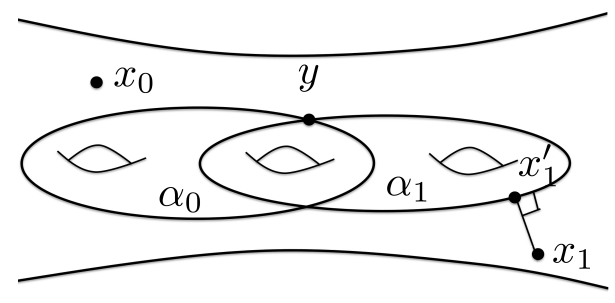

Figure 2. The case where $\alpha_{0} \cap \alpha_{1} \neq \emptyset$.

holds (cf. $\S 4.1$ or $\S 5.2$ of [4]). Since $\ell_{R_{0}}\left(\left[c_{x}\right]\right)>1 / M$ and $\ell_{R_{0}}(\zeta) \leq \ell_{R_{0}}\left(c_{x}\right)<M$,

$$
\cosh r_{x}=\frac{\ell_{R_{0}}(\zeta)}{\ell_{R_{0}}\left(\left[c_{x}\right]\right)}<M^{2} .
$$

Since $e^{r_{x}} / 2<\cosh r_{x}$ holds, $e^{r_{x}}<2 M^{2}$, that is, $r_{x}<2 \log (\sqrt{2} M)$. Therefore $d_{R_{0}}\left(\left[c_{x}\right], x\right) \leq r_{x}+d_{R_{0}}(\zeta, x)<r_{x}+1 / 2 \cdot \ell_{R_{0}}\left(c_{x}\right)<2 \log (\sqrt{2} M)+1 / 2 M$.

Put

$$
d=\sup _{x \in R_{0}^{\prime}} d_{x}
$$

Then $d=d(M)$ is a constant such that for any $x \in R_{0}^{\prime}$, there exists a closed geodesic $\alpha_{x}$ satisfying $\ell_{R_{0}}\left(\alpha_{x}\right)<M$ and $d_{R_{0}}\left(x, \alpha_{x}\right)<d$. Indeed, for an arbitrary $x \in R_{0}^{\prime}$, let $c_{x}$ be an arbitrary simple closed curve based on $x$ with $1 / M<\ell_{R_{0}}\left(c_{x}\right)<M$. Then $\ell_{R_{0}}\left(\left[c_{x}\right]\right)<M$ and $d_{R_{0}}\left(\left[c_{x}\right], x\right) \leq d_{x}<d$. Put

$$
D=2 \max \{M, d\} \text {. }
$$

By using constants $M, d, D$, we shall take pairwise disjoint simple closed geodesics $\mathcal{G}=\left\{\alpha_{n}\right\}_{n=0}^{\infty}$. Let $x_{0}$ be an arbitrary point in $R_{0}^{\prime}$ and take a closed geodesic $\alpha_{0}$ with the length $<M$ and with $d_{R_{0}}\left(\alpha_{0}, x_{0}\right)<d$. Let $U_{0}$ be the $D$-neighborhood of $\alpha_{0}$, i.e.,

$$
U_{0}:=B\left(\alpha_{0}, D\right)=\left\{x \in R_{0}^{\prime} \mid d_{R_{0}}\left(\alpha_{0}, x\right)<D\right\} .
$$

Now take a point $x_{1} \in \partial U_{0}$. And for $x_{1}$, take a simple closed geodesic $\alpha_{1}$ with $\ell_{R_{0}}\left(\alpha_{1}\right)<M$ and with $d_{R_{0}}\left(x_{1}, \alpha_{1}\right)<d$. Then:

Claim 2.3. $\alpha_{0} \cap \alpha_{1}=\emptyset$ and $U_{0} \cap B\left(\alpha_{1}, D\right) \neq \emptyset$, where $B\left(\alpha_{1}, D\right)$ is the $D$ neighborhood of $\alpha_{1}$ in $R_{0}^{\prime}$.

Proof. Assume that $\alpha_{0} \cap \alpha_{1} \neq \emptyset$ and take a point $y \in \alpha_{0} \cap \alpha_{1}$. Now let $x_{1}^{\prime}$ be the point on $\alpha_{1}$ that is closest to $x_{1}$. (See Figure 2.) 
Since $y \in \alpha_{1}$,

$$
\begin{aligned}
d_{R_{0}}\left(y, x_{1}\right) & <d_{R_{0}}\left(y, x_{1}^{\prime}\right)+d_{R_{0}}\left(x_{1}^{\prime}, x_{1}\right) \\
& \leq \frac{1}{2} \ell_{R_{0}}\left(\alpha_{1}\right)+d_{R_{0}}\left(x_{1}^{\prime}, x_{1}\right) \\
& <\frac{1}{2} M+d \\
& <\frac{3}{2} \max \{M, d\} .
\end{aligned}
$$

On the other hand, $d_{R_{0}}\left(\alpha_{0}, x_{1}\right)<d_{R_{0}}\left(y, x_{1}\right)$ holds since $y \in \alpha_{0}$. Hence

$$
d_{R_{0}}\left(\alpha_{0}, x_{1}\right)<\frac{3}{2} \max \{M, d\}<D .
$$

This contradicts the assumption that $x_{1} \in \partial U_{0}$, that is, $d_{R_{0}}\left(\alpha_{0}, x_{1}\right)=D$. Therefore $\alpha_{0} \cap \alpha_{1}=\emptyset$.

Next we consider the $d$-neighborhood $B\left(x_{1}, d\right)$ of $x_{1}$. Since $x_{1}$ a point in $\partial U_{0}$, $U_{0} \cap B\left(x_{1}, d\right) \neq \emptyset$. For any $x \in U_{0} \cap B\left(x_{1}, d\right)$,

$$
d_{R_{0}}\left(x, \alpha_{1}\right)<d_{R_{0}}\left(x, x_{1}\right)+d_{R_{0}}\left(x_{1}, \alpha_{1}\right)<d+d \leq D .
$$

Hence $x \in U_{0}$ and $x \in B\left(\alpha_{1}, D\right)$. Therefore $U_{0} \cap B\left(\alpha_{1}, D\right) \neq \emptyset$.

Next take an arbitrary point $x_{2}$ in $\partial U_{0}-B\left(\alpha_{1}, D\right)$. Also take a simple closed geodesic $\alpha_{2}$ such that $\ell_{R_{0}}\left(\alpha_{2}\right)<M$ and $d_{R_{0}}\left(x_{2}, \alpha_{2}\right)<d$. We can check that $\alpha_{0}, \alpha_{1}$ and $\alpha_{2}$ are disjoint and $U_{0} \cap B\left(\alpha_{2}, D\right) \neq \emptyset$ since $d_{R_{0}}\left(\alpha_{0}, x_{2}\right)=D$ and $d_{R_{0}}\left(\alpha_{1}, x_{2}\right) \geq D$. Continue to take a point in $\partial U_{0}-\bigcup_{i} B\left(\alpha_{i}, D\right)$ and a geodesic until $\partial U_{0} \subset \bigcup_{i=1}^{n(1)} B\left(\alpha_{i}, D\right)$.

After that, put

$$
U_{1}:=U_{0} \cup\left(\bigcup_{i=1}^{n(1)} B\left(\alpha_{i}, D\right)\right) .
$$

Next, for this, take a point $x_{n(1)+1} \in \partial U_{1}$, and take a simple closed geodesic $\alpha_{n(1)+1}$ such that $\ell_{R_{0}}\left(\alpha_{n(1)+1}\right)<M$ and $d_{R_{0}}\left(\alpha_{n(1)+1}, x_{n(1)+1}\right)<d$. Then $\alpha_{0}, \alpha_{1}, \ldots, \alpha_{n(1)}$ and $\alpha_{n(1)+1}$ are disjoint and $U_{1} \cap B\left(\alpha_{n(1)+1}, D\right) \neq \emptyset$. Similarly, take points and closed geodesics $\alpha_{n(1)+2}, \ldots, \alpha_{n(2)}$ until $\partial U_{1} \subset \bigcup_{i=n(1)+1}^{n(2)} B\left(\alpha_{i}, D\right)$. And put

$$
U_{2}:=U_{1} \cup\left(\bigcup_{i=n(1)+1}^{n(2)} B\left(\alpha_{i}, D\right)\right) .
$$

Continue to take a union $U_{L}$ of the $D$-neighborhoods of geodesics such that $U_{0} \subset U_{1} \subset U_{2} \subset \cdots \subset U_{L} \subset R_{0}^{\prime}$. Then, for any closed geodesic $\alpha_{n}$ taken in $U_{L}$, the length $<M$ holds, and for any $x \in U_{L}, d_{R_{0}}\left(x, \alpha_{i}\right)<D$ for some $\alpha_{i} \in\left\{\alpha_{n}\right\}_{n=0}^{n(L)}$ since $x \in B\left(\alpha_{i}, D\right)$ for some $\alpha_{i} \in\left\{\alpha_{n}\right\}_{n=0}^{n(L)}$. Put $U:=\lim _{L \rightarrow \infty} U_{L}$, then $U=R_{0}^{\prime}$. Indeed, $U \subset R_{0}^{\prime}$ is trivial. Assume that $R_{0}^{\prime} \not \subset U$. For any $x \in R_{0}^{\prime}-U$, take the shortest geodesic segment $s$ from $x$ to $\alpha_{0}$ (the first closed geodesic in $U_{0}$ ). Then there exists a point $y \in s \cap \partial U$. Since $U$ is a union of neighborhoods $\left\{B\left(\alpha_{i}, D\right)\right\}$ of closed geodesics, there exists a number $i$ such that $y \in \partial B\left(\alpha_{i}, D\right)$. However, for any $i, \partial B\left(\alpha_{i}, D\right)$ is covered by some neighborhoods $\left\{B\left(\alpha_{j}, D\right)\right\}$ by construction. This is a contradiction. Hence $R_{0}^{\prime} \subset U$. Therefore, we obtain simple closed geodesics $\mathcal{G}=\left\{\alpha_{n}\right\}_{n=0}^{\infty}$ as desired. 
By using Lemma 2.1, we prove the Key Lemma.

Proof of Lemma 1.8. Let $\mathcal{G}=\left\{\alpha_{n}\right\}_{n=0}^{\infty}$ be the closed geodesics in Lemma 2.1] For each $\alpha_{n} \in \mathcal{G}$, take the following domain:

$$
D_{n}:=\left\{x \in R_{0}^{\prime} \mid d_{R_{0}}\left(x, \alpha_{n}\right)<d_{R_{0}}\left(x, \alpha_{\ell}\right) \text { for any } \ell \neq n\right\} .
$$

We call $D_{n}$ a Voronoi domain of $\alpha_{n}$. Note that for any point $x \in \partial D_{n}, d_{R_{0}}\left(x, \alpha_{n}\right) \leq$ $D$ holds by condition (2) in Lemma 2.1 Also, for any point $x \in \partial D_{n}$, there exists a Voronoi domain $D_{m}$ of $\alpha_{m}\left(\neq \alpha_{n}\right)$ such that $x \in \partial D_{m}$ and $d_{R_{0}}\left(x, \alpha_{n}\right)=d_{R_{0}}\left(x, \alpha_{m}\right)$. Then $d_{R_{0}}\left(\alpha_{n}, \alpha_{m}\right) \leq 2 D$.

We shall consider $D_{n}$ and a domain $\left[D_{n}\right]$ obtained by straightening each component of $\partial D_{n}$ for each $n$. (We assume that any component of $\partial D_{n}$ is simple. If some component has self-intersection points, divide it into simple closed curves.) We describe how to take $\left[D_{n}\right]$ : If $c \cap \partial D_{n}=\emptyset$ for any horocycle $c$ bounding a cusp neighborhood of $R_{0}$, let $\left[D_{n}\right] \subset R_{0}^{\prime}$ be a domain obtained by replacing each component $\sigma_{n}$ of $\partial D_{n}$ with the closed geodesic $\left[\sigma_{n}\right]$ homotopic to $\sigma_{n}$. Then note the following.

Claim 2.4. There exists a constant $E=E(M)$ such that for any $x \in\left[\sigma_{n}\right], d_{R_{0}}\left(\alpha_{n}, x\right)$ $\leq E(n=0,1,2 \ldots)$.

Proof. First, note that there exists a point $x_{0} \in\left[\sigma_{n}\right]$ such that $d_{R_{0}}\left(\alpha_{n}, x_{0}\right)<2 D$ holds. Indeed, for $\sigma_{n}$, there exists a Voronoi domain $D_{m}$ of $\alpha_{m}\left(\neq \alpha_{n}\right)$ such that $\sigma_{n} \cap \partial D_{m} \neq \emptyset$. Let $s_{n m}$ be the shortest geodesic segment connecting $\alpha_{n}$ and $\alpha_{m}$, then $\ell_{R_{0}}\left(s_{n m}\right)<2 D$. Any closed curve homotopic to $\sigma_{n}$ intersects $s_{n m}$, therefore there exists a point $x_{0} \in\left[\sigma_{n}\right] \cap s_{n m}$.

Next we take a constant $E$. In the unit disk $\mathbb{D}$, take an arbitrary segment $\alpha$ with the hyperbolic length $M$ and let $L$ be the length of the boundary $\partial B(\alpha, 2 D)$ of the $2 D$-neighborhood of $\alpha$. We put $E:=2 D+\frac{1}{2} L$.

Now we assume that there exists a point $x_{1} \in\left[\sigma_{n}\right]$ such that $d_{R_{0}}\left(\alpha_{n}, x_{1}\right)>E$. By existence of $x_{0}$ and $x_{1}$, we can take some points $x_{2}, x_{3} \in\left[\sigma_{n}\right]$ around $x_{1}$ such that $d_{R_{0}}\left(\alpha_{n}, x_{i}\right)=2 D(i=2,3)$ and they are put on $\left[\sigma_{n}\right]$ in the order of $x_{2}, x_{1}, x_{3}$. Here, let $S$ be the set surrounded by $\sigma_{n}$ and $\left[\sigma_{n}\right]$, then $S$ has genus zero and no horocycles (bounding cusp neighborhoods). In $S$, we can take an arc $c\left[x_{2}, x_{3}\right]$ such that its endpoints are $x_{2}$ and $x_{3}$, and $d_{R_{0}}\left(x, \alpha_{n}\right)=2 D$ for any $x \in c\left[x_{2}, x_{3}\right]$ since $d_{R_{0}}\left(y, \alpha_{n}\right) \leq D$ for any $y \in \sigma_{n}$. Then $\ell_{R_{0}}\left(c\left[x_{2}, x_{3}\right]\right)<L$ since $\ell_{R_{0}}\left(\alpha_{n}\right)<M$. On the other hand, let $g\left[x_{i}, x_{j}\right]$ be the geodesic segment on $\left[\sigma_{n}\right]$ such that its endpoints are $x_{i}$ and $x_{j}$, and satisfies

$$
\ell_{R_{0}}\left(g\left[x_{2}, x_{3}\right]\right)=\ell_{R_{0}}\left(g\left[x_{2}, x_{1}\right]\right)+\ell_{R_{0}}\left(g\left[x_{1}, x_{3}\right]\right) .
$$

Since $d_{R_{0}}\left(\alpha_{n}, x_{1}\right)>E=2 D+\frac{1}{2} L$ and $d_{R_{0}}\left(\alpha_{n}, x_{i}\right)=2 D(i=2,3)$,

$$
d_{R_{0}}\left(x_{1}, x_{i}\right) \geq d_{R_{0}}\left(\alpha_{n}, x_{1}\right)-d_{R_{0}}\left(\alpha_{n}, x_{i}\right)>2 D+\frac{1}{2} L-2 D=\frac{1}{2} L
$$

$(i=2,3)$. Hence

$$
\ell_{R_{0}}\left(g\left[x_{2}, x_{3}\right]\right)>\frac{1}{2} L+\frac{1}{2} L=L ;
$$

that is, $\ell_{R_{0}}\left(c\left[x_{2}, x_{3}\right]\right)<L<\ell_{R_{0}}\left(g\left[x_{2}, x_{3}\right]\right)$. This is a contradiction.

Next we consider the case where there exist a horocycle $c$ bounding a cusp neighborhood and a domain $D_{n}$ such that $c \cap \partial D_{n} \neq \emptyset$. We do not want to leave $R_{0}-\cup_{n}\left[D_{n}\right]$ having punctures, so we take $\left[D_{n}\right]$ as follows: For a horocycle $c$, let 
$\left\{D_{i}\right\}_{i \in I}$ be all Voronoi domains with $\partial D_{i} \cap c \neq \emptyset$ and take an arbitrary $D_{n}$ in $\left\{D_{i}\right\}_{i \in I}$. For $D_{n}$, let $\sigma_{n}$ be a component of $\partial D_{n}$ with $c \cap \sigma_{n} \neq \emptyset$. Let $c_{n}$ denote the intersection of $c$ with the interior of $D_{n}$ and put $\sigma_{n}^{\prime}=\left(\left(\sigma_{n} \cap R_{0}^{\prime}\right) \cup c\right)-c_{n}$. (See Figure 3.) And take a closed geodesic $\left[\sigma_{n}^{\prime}\right]$ as a boundary component of $\left[D_{n}\right]$. On the other hand, for the other Voronoi domain $D_{m}(m \in I-\{n\})$, take a closed geodesic $\left[\sigma_{m}\right]$ homotopic to an original boundary component $\sigma_{m}$ (i.e., a curve without adding or removing subhorocycles of $c$ ) as a boundary component of $\left[D_{m}\right]$. Note that for any $x \in\left[\sigma_{n}^{\prime}\right]$ (or $\left.x \in\left[\sigma_{m}\right]\right), d_{R_{0}}\left(\alpha_{n}, x\right)<E\left(\right.$ or $d_{R_{0}}\left(\alpha_{m}, x\right)<E$ ) for the same constant $E=E(M)$ as in Claim 2.4 since $d_{R_{0}}\left(\alpha_{n}, \alpha_{m}\right) \leq 2 D$.

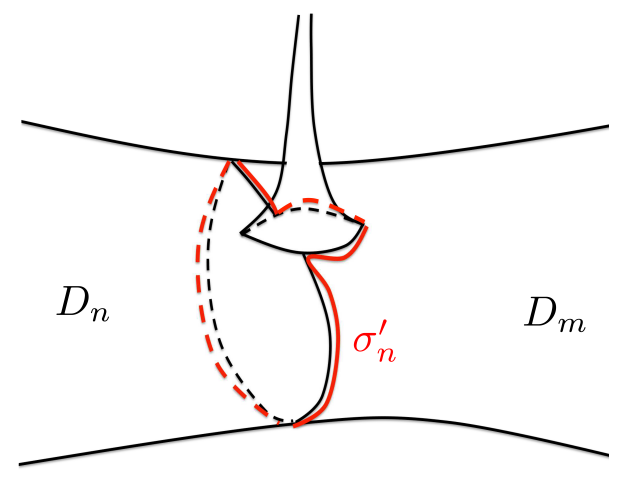

FIGURE 3. $\sigma_{n}^{\prime}$

Now we consider pants decomposition in each $\left[D_{n}\right]$. First, let us check that genus $g_{n}$ of $\left[D_{n}\right]$ and the number of connected components of $\partial\left[D_{n}\right]$ are uniformly bounded. For each $\left[D_{n}\right]$, let $h_{n}$ be the number of horocycles bounding cusp neighborhoods and $b_{n}$ be the number of closed geodesics of $\partial\left[D_{n}\right]$.

Claim 2.5. There exists a constant $A=A(M)>0$ such that $g_{n}<A, h_{n}<A$ and $b_{n}<A$ for any $\left[D_{n}\right]$.

Proof. In the unit disk $\mathbb{D}$, consider an arbitrary geodesic segment $\alpha$ with the hyperbolic length $M$. And let $A$ be the area of the $E$-neighborhood of $\alpha$. Then (the area of $\left.\left[D_{n}\right]\right)<A$, since $\left[D_{n}\right]$ is contained in the $E$-neighborhood of $\alpha_{n}$.

Now let $\left[\hat{D_{n}}\right]$ be a surface obtained by adding original cusp neighborhoods of $R_{0}$ to all horocycles. Then, since a cusp neighborhood has area one, (the area of $\left.\left[\hat{D_{n}}\right]\right)=\left(\right.$ the area of $\left.\left[D_{n}\right]\right)+h_{n}<A+h_{n}$.

On the other hand, $\left[\hat{D}_{n}\right]$ is of genus $g_{n}$ and with $h_{n}$ punctures and $b_{n}$ boundary geodesics, so, by the theorem of Gauss-Bonnet,

$$
\text { (The area of } \left.\left[\hat{D}_{n}\right]\right)=2 \pi\left(2 g_{n}-2+h_{n}+b_{n}\right)<A+h_{n} \text {. }
$$

Therefore

$$
4 \pi g_{n}+(2 \pi-1) h_{n}+2 \pi b_{n}<A+4 \pi .
$$

Put $A:=A+4 \pi$, then $g_{n}<A, h_{n}<A$ and $b_{n}<A$ hold.

Also, we check the following:

Claim 2.6. There exists a constant $B=B(M)>0$ such that $\ell_{R_{0}}\left(\left[\sigma_{n}\right]\right)<B$ for any $\left[D_{n}\right]$ and any component $\left[\sigma_{n}\right]$ of $\partial\left[D_{n}\right]$. 
Proof. In the unit disk $\mathbb{D}$, take an arbitrary segment $\alpha$ with the hyperbolic length $M$ and let $K$ be the length of the boundary $\partial B(\alpha, E)$ of the $E$-neighborhood of $\alpha$. Put $B:=2(K+E)$.

Now, for $\left[\sigma_{n}\right]$ and $\alpha_{n}$, take the shortest geodesic segment $s$ connecting them, and let $x \in\left[\sigma_{n}\right]$ be the endpoint of $s$, and let $\ell$ be the length of $s$. Then $\ell \leq E$. Let $y$ be the point on $\left[\sigma_{n}\right]$ such that $d_{R_{0}}(x, y)=\frac{1}{2} \ell_{R_{0}}\left(\left[\sigma_{n}\right]\right)$, and let $\frac{1}{2}\left[\sigma_{n}\right]$ be the half of $\left[\sigma_{n}\right]$ with endpoints $x$ and $y$. Note that $\frac{1}{2}\left[\sigma_{n}\right]$ is the shortest geodesic segment between $x$ and $y$ since $\left[D_{n}\right]$ is convex by the definition. Also, take the shortest geodesic segment $s^{\prime}$ from $y$ to $\alpha_{n}$. Note that $\ell \leq \ell_{R_{0}}\left(s^{\prime}\right) \leq E$. Now, on $s^{\prime}$, take the point $z$ such that $d_{R_{0}}\left(z, \alpha_{n}\right)=\ell$ and take the shortest geodesic segment $s_{1}$ from $x$ to $z$. And let $s_{2}$ be the shortest geodesic segment from $z$ to $y$. Then

$$
\ell_{R_{0}}\left(\frac{1}{2}\left[\sigma_{n}\right]\right) \leq \ell_{R_{0}}\left(s_{1}\right)+\ell_{R_{0}}\left(s_{2}\right)
$$

holds. Since $\ell_{R_{0}}\left(s_{2}\right)<E$ and we see that $\ell_{R_{0}}\left(s_{1}\right)<K$, hence $\frac{1}{2} \ell_{R_{0}}\left(\left[\sigma_{n}\right]\right)=$ $\ell_{R_{0}}\left(\frac{1}{2}\left[\sigma_{n}\right]\right)<K+E=\frac{1}{2} B$.

From Claims 2.5 and 2.6 and Bers' constant proved by Farb and Margalit ([7], $\S 12.4 .2)$ or Buser $(4], \S 5.2)$, we obtain the following:

Claim 2.7. There exists a constant $F=F(M)>0$ and pairs of pants $\left\{P_{k}\right\}$ such that $\left[\hat{D_{n}}\right]=\bigcup_{k} P_{k}$, and for any closed geodesic $\gamma_{i}$ in $\partial P_{k}, \ell_{R_{0}}\left(\gamma_{i}\right)<F(i=$ $1, \ldots, 3 g_{n}-3+h_{n}+b_{n}$ ) for each $\left[\hat{D_{n}}\right]$.

Let $\mathcal{P}=\left\{P_{k}\right\}_{k=0}^{\infty}$ be a family of all pairs of pants in all domains $\left\{\left[\hat{D}_{n}\right]\right\}_{n=0}^{\infty}$. Finally we take hexagons in $R_{0}-\bigcup_{k=0}^{\infty} P_{k}$. Let $\mathcal{D}=\left\{D_{n}\right\}_{n=0}^{\infty}$ be all Voronoi domains and take an arbitrary domain $D_{0} \in \mathcal{D}$. Let $D_{1} \in \mathcal{D}$ be a domain with $\partial D_{0} \cap \partial D_{1} \neq \emptyset$.

Case I: Suppose $\partial D_{2} \cap\left(\partial D_{0} \cap \partial D_{1}\right)=\emptyset$ for any $D_{2} \in \mathcal{D}$.

By construction, there exist two pairs of pants $P_{0} \subset\left[D_{0}\right]$ and $P_{1} \subset\left[D_{1}\right]$ with $\partial P_{0} \cap \partial P_{1} \neq \emptyset$, that is, $D_{0} \cup D_{1}$ is connected, hence we do not take a hexagon. (See Figure 4)

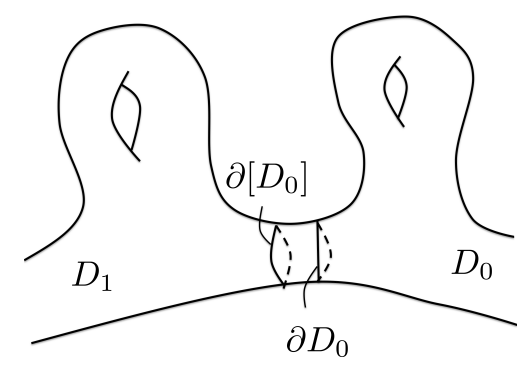

Figure 4. Case I

Case II: Suppose $\partial D_{2} \cap\left(\partial D_{0} \cap \partial D_{1}\right) \neq \emptyset$ for some $D_{2} \in \mathcal{D}$.

Note that $\partial D_{2} \cap\left(\partial D_{0} \cap \partial D_{1}\right)$ consists of a point or finite points. Take a point $v \in$ $\partial D_{2} \cap\left(\partial D_{0} \cap \partial D_{1}\right)$ and let $\left\{D_{0}, D_{1}, \ldots, D_{\ell}\right\} \subset \mathcal{D}$ be a set of all domains each of whose boundary contains $v$ and which is in counterclockwise direction around $v$. Connect $v$ to $\partial\left[D_{i}\right]$ with the shortest segment $s_{i}$ for each $i=0,1, \ldots, \ell$. (See Figure 5.) Regard $s_{i} \cdot s_{i+1}$ as an arc connecting $\partial\left[D_{i}\right]$ and $\partial\left[D_{i+1}\right]$, and take the shortest geodesic 
$\left[s_{i} \cdot s_{i+1}\right]$ homotopic to $s_{i} \cdot s_{i+1}$ for each $i$, where the homotopy glides endpoints on each closed geodesic. Then we get a $2(\ell+1)$-sided right polygon. If $\ell \geq 3$, take the geodesics $\left[s_{0} \cdot s_{2}\right], \ldots,\left[s_{0} \cdot s_{\ell-1}\right]$. Then we get $(\ell-1)$ right hexagons. The length of each edge is bounded by $2 E$ since $\ell_{R_{0}}\left(s_{i}\right)<\max \left\{d_{R_{0}}\left(\partial D_{i}, \alpha_{i}\right), d_{R_{0}}\left(\partial\left[D_{i}\right], \alpha_{i}\right)\right\} \leq E$ for each $i$.

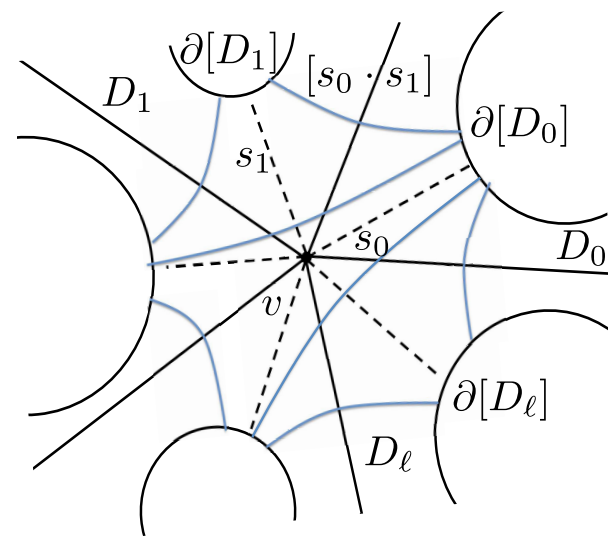

FiguRE 5. Right hexagons around $v$

Take hexagons for each intersection of boundaries of $\left\{D_{n}\right\}$. Then a family of hexagons $\left\{H_{m}\right\}_{m=1}^{\infty}$ we desire is obtained.

\section{Proof of Theorem 1.7}

Before starting to prove Theorem 1.7 we introduce Bishop's lemmas on quasiconformal mappings.

Lemma 3.1 (Bishop [3], Lemma 3.1). Let $T_{1}, T_{2} \subset \mathbb{D}$ be two hyperbolic triangles with sides $\left(a_{1}, b_{1}, c_{1}\right)$ and $\left(a_{2}, b_{2}, c_{2}\right)$, respectively. Suppose all their angles are bounded below by $\theta>0$ and

$$
\varepsilon:=\max \left(\left|\log \frac{a_{1}}{a_{2}}\right|,\left|\log \frac{b_{1}}{b_{2}}\right|,\left|\log \frac{c_{1}}{c_{2}}\right|\right) \leq A .
$$

Then there is a constant $C=C(\theta, A)>0$ and $a(1+C \varepsilon)$-quasiconformal mapping $\varphi: T_{1} \rightarrow T_{2}$ such that $\varphi$ maps each vertex to the corresponding vertex and $\varphi$ is affine on each edge of $T_{1}$.

Lemma 3.2 (Bishop [3], Corollary 3.3). Let $H, H^{\prime} \subset \mathbb{D}$ be two hyperbolic hexagons with sides $\left(a_{1}, \ldots, a_{6}\right)$ and $\left(b_{1}, \ldots, b_{6}\right)$, respectively. Suppose $a_{1}, \ldots, a_{6}$ and $b_{1}, \ldots, b_{6}$ are bounded above by a constant $B$ and below by $1 / B$. Also assume that three alternating angles of $H$ and the corresponding angles of $H^{\prime}$ are $\pi / 2$ and the remaining angles are bounded below by $\theta>0$ and above by $\pi-\theta$. If $\varepsilon=\max _{i}\left|\log a_{i} / b_{i}\right| \leq 2$, then there is a constant $C=C(\theta, B)>0$ and $a(1+C \varepsilon)$-quasiconformal mapping $\varphi: H \rightarrow H^{\prime}$ such that $\varphi$ maps each vertex to the corresponding vertex and $\varphi$ is affine on each edge of $H$.

Let us start the proof of Theorem 1.7 For a sufficiently small number $r>0$ and a basepoint $p_{0}=\left[R_{0}, i d\right]$, put $B\left(p_{0}, r\right)=\left\{p \in T\left(R_{0}\right) \mid d_{L}\left(p_{0}, p\right)<r\right\}$. For 
any $p_{1}, p_{2} \in B\left(p_{0}, r\right)$, we show that $\frac{1}{C} d_{T}\left(p_{1}, p_{2}\right) \leq d_{L}\left(p_{1}, p_{2}\right)$. Put $p_{1}:=\left[R_{1}, f_{1}\right]$, $p_{2}:=\left[R_{2}, f_{2}\right]$ and $g:=f_{2} \circ f_{1}^{-1}$.

Since $R_{0}$ has bounded geometry for a constant $M, R_{1}$ has bounded geometry for some constant $M_{1}$ depending on $M$ and $r$. Hence $R_{1}$ can be decomposed into pairs of pants $\mathcal{P}_{1}$ and right hexagons $\mathcal{H}_{1}$ by Lemma 1.8. First, let us consider the corresponding decomposition in $R_{2}$. Note that $d_{L}\left(p_{1}, p_{2}\right)<2 r$, thus

$$
\left|\log \frac{\ell_{R_{1}}([\alpha])}{\ell_{R_{2}}([g(\alpha)])}\right|<2 r
$$

holds for any $\alpha \in \mathcal{C}\left(R_{1}\right)$. For an arbitrary hexagon $H \in \mathcal{H}_{1}$, let $c_{1}, c_{2}, c_{3}$ be connected components of boundaries of three pairs of pants $P_{1}, P_{2}, P_{3} \in \mathcal{P}_{1}$ around $H$. Let $e_{1}, \ldots, e_{6}$ be the edges of $H$ (in counterclockwise direction), where $e_{2}$ is the segment connecting $c_{1}$ and $c_{2}$. (See Figure [6.) Take a closed curve $c_{12}:=$ $c_{1} \cdot e_{2} \cdot c_{2} \cdot e_{2}^{-1}$ in $R_{1}$. For $g\left(c_{12}\right)$ in $R_{2}$, take a closed geodesic $\left[g\left(c_{12}\right)\right]$ and consider a pair of pants with boundary $\left\{\left[g\left(c_{1}\right)\right],\left[g\left(c_{2}\right)\right],\left[g\left(c_{12}\right)\right]\right\}$. Let $e_{2}^{\prime}$ be the geodesic orthogonal to $\left[g\left(c_{1}\right)\right]$ and $\left[g\left(c_{2}\right)\right]$ in the pants. Since the lengths of $e_{2}$ and $e_{2}^{\prime}$ are determined by the lengths of $\left\{c_{1}, c_{2}, c_{12}\right\}$ and $\left\{\left[g\left(c_{1}\right)\right],\left[g\left(c_{2}\right)\right],\left[g\left(c_{12}\right)\right]\right\}$, respectively,

$$
\frac{1}{A}<\frac{\ell_{R_{1}}\left(e_{2}\right)}{\ell_{R_{2}}\left(e_{2}^{\prime}\right)}<A
$$

holds, where $A$ is a constant depending on $r$ and $M$. Similarly, take segments $e_{4}^{\prime}, e_{6}^{\prime}$ for $e_{4}, e_{6}$, respectively. Let $H^{\prime}$ be a right hexagon with edges $e_{2}^{\prime}, e_{4}^{\prime}, e_{6}^{\prime}$ and subarcs $e_{1}^{\prime}, e_{3}^{\prime}, e_{5}^{\prime}$ of $\left[g\left(c_{1}\right)\right],\left[g\left(c_{2}\right)\right],\left[g\left(c_{3}\right)\right]$ in $R_{2}$. Then

$$
\frac{1}{B}<\frac{\ell_{R_{1}}\left(e_{i}\right)}{\ell_{R_{2}}\left(e_{i}^{\prime}\right)}<B
$$

$(i=1,3,5)$ holds, where $B$ is a constant depending on $r$ and $M$, since a right hexagon is determined by the lengths of three alternating edges. For each hexagon in $R_{1}$, take a hexagon in $R_{2}$ in the above way; then we have a similar family of hexagons $\mathcal{H}_{2} \subset R_{2}$. And we have a quasiconformal map $\varphi$ from $\mathcal{H}_{1}$ to $\mathcal{H}_{2}$ by Lemma 3.2

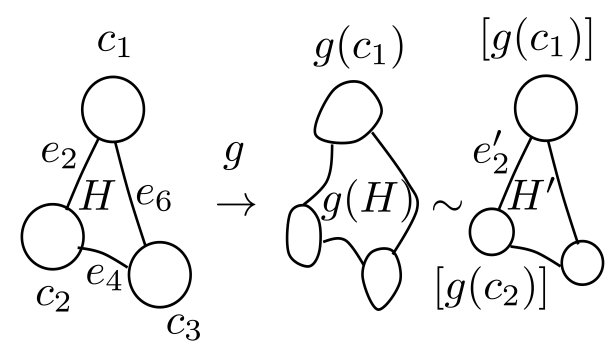

Figure $6 . H \in \mathcal{H}_{1}$ and $H^{\prime} \in \mathcal{H}_{2}$.

Next we consider the quasiconformal map on $\mathcal{P}_{1}$. Let $P_{n}$ be an arbitrary pair of pants in $\mathcal{P}_{1}$ and $\left\{\alpha_{1}, \alpha_{2}, \alpha_{3}\right\}$ be boundary components of $P_{n}$. (If some $\alpha_{i}$ is a puncture, replace it with a horocycle.) We consider triangulation of $P_{n}$. First, take a subset $X_{j}=\left\{x_{i}\right\}$ of $\alpha_{j}(j=1,2,3)$ consisting of vertices of hexagons $\left\{H_{m}\right\}$ with $\alpha_{j} \cap H_{m} \neq \emptyset$. If there exists a closed geodesic $\alpha_{j}$ such that $\alpha_{j} \cap H_{m}=\emptyset$ for any $H_{m} \in \mathcal{H}_{1}$, then $X_{j}=\emptyset$. Also, decompose $P_{n}$ into two symmetric right hexagons 
$\left\{h_{1}, h_{2}\right\}$ and let $Y=\left\{y_{1}, \ldots, y_{6}\right\}$ (counterclockwise direction) be a subset of $\partial P_{n}$ consisting of the vertices of $\left\{h_{1}, h_{2}\right\}$, where the segment $\left[y_{1}, y_{2}\right]$ are connecting $\alpha_{3}$ and $\alpha_{1}$, and $\left[y_{2}, y_{3}\right] \subset \alpha_{1}$. (See Figure 7), Now let $w$ be a midpoint of $\left[y_{1}, y_{2}\right]$ and put $Z:=X_{1} \cup X_{2} \cup X_{3} \cup Y$. Connect $w$ to each point of $Z$ by the geodesic segment $\left[w, x_{i}\right]$ or $\left[w, y_{k}\right]$; then we obtain triangulation of $P_{n}$.
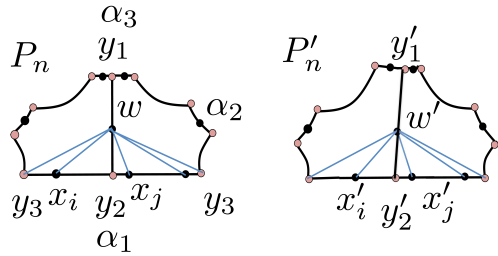

FiguRe 7. $Z \subset \partial P_{n}$ and $Z^{\prime} \subset \partial P_{n}^{\prime}$.

Second, we consider triangulation of pairs of pants in $R_{2}$. Let $P_{n}^{\prime} \in \mathcal{P}_{2}$ be a pair of pants with boundary $\left\{\left[g\left(\alpha_{1}\right)\right],\left[g\left(\alpha_{2}\right)\right],\left[g\left(\alpha_{3}\right)\right]\right\}$. Let $X_{j}^{\prime}=\left\{x_{i}^{\prime}\right\}(j=1,2,3)$ be a subset of $\left[g\left(\alpha_{j}\right)\right]$ such that each point $x_{i}^{\prime}$ is the vertex of $H_{m}^{\prime} \in \mathcal{H}_{2}$ corresponding to $x_{i} \in$ $R_{1}$. If $X_{j}=\emptyset$, then $X_{j}^{\prime}=\emptyset$. Also, we take points $Y^{\prime}$ corresponding $Y$ as follows. If $X_{j}^{\prime} \neq \emptyset$, let $y_{k}^{\prime}$ be the point satisfying $\ell_{R_{1}}\left[x_{i}, y_{k}\right] / \ell_{R_{1}}\left[x_{i}, x_{j}\right]=\ell_{R_{2}}\left[x_{i}^{\prime}, y_{k}^{\prime}\right] / \ell_{R_{2}}\left[x_{i}^{\prime}, x_{j}^{\prime}\right]$, where $[\cdot, \star]$ is a segment with endpoints $\cdot$ and $\star$, and $\left[x_{i}, x_{j}\right],\left[x_{i}^{\prime}, x_{j}^{\prime}\right]$ are the smallest segments including $y_{k}$ and $y_{k}^{\prime}$, respectively. If $X_{j}^{\prime}=\emptyset$, let $y_{k}^{\prime}$ be the point on $\left[g\left(\alpha_{j}\right)\right]$ which is one of vertices of two symmetric right hexagons $\left\{h_{1}^{\prime}, h_{2}^{\prime}\right\}$ decomposing $P_{n}^{\prime}$. As above, we assume that the segment $\left[y_{1}^{\prime}, y_{2}^{\prime}\right]$ are connecting $\left[g\left(\alpha_{3}\right)\right]$ and $\left[g\left(\alpha_{1}\right)\right]$, and $\left[y_{2}^{\prime}, y_{3}^{\prime}\right] \subset\left[g\left(\alpha_{1}\right)\right]$. Let $w^{\prime}$ be a midpoint of $\left[y_{1}^{\prime}, y_{2}^{\prime}\right]$ and put $Z^{\prime}:=X_{1}^{\prime} \cup X_{2}^{\prime} \cup X_{3}^{\prime} \cup Y^{\prime}$. Connect $w^{\prime}$ to each point of $Z^{\prime}$ by the geodesic segment $\left[w^{\prime}, x_{i}^{\prime}\right]$ or $\left[w^{\prime}, y_{k}^{\prime}\right]$; then we obtain triangulation of $P_{n}^{\prime}$. Note the lengths of the corresponding sides of triangles.

Claim 3.3. There exists a constant $D=D(r, M)$ such that for any $i$ and $k$,

$$
\frac{1}{D}<\frac{\ell_{R_{1}}\left(\left[w, x_{i}\right]\right)}{\ell_{R_{2}}\left(\left[w^{\prime}, x_{i}^{\prime}\right]\right)}<D \text { and } \frac{1}{D}<\frac{\ell_{R_{1}}\left(\left[w, y_{k}\right]\right)}{\ell_{R_{2}}\left(\left[w^{\prime}, y_{k}^{\prime}\right]\right)}<D
$$

hold.

Proof. For $P_{n}$, take the nearest pair of pants $P_{\ell} \in \mathcal{P}_{1}-\left\{P_{n}\right\}$ to $\alpha_{1}$ such that $\partial P_{n} \cap \partial P_{\ell}=\emptyset$. Also take the shortest arc $\beta$ connecting $\alpha_{1}$ and a component $\alpha_{\ell}$ of $\partial P_{\ell}$, and let $P$ be a pair of pants obtained by $\alpha_{1}, \alpha_{\ell}$ and $\alpha_{1} \cdot \beta \cdot \alpha_{\ell} \cdot \beta^{-1}$. (See Figure 8.) Put $S_{1}:=P \cup P_{n} \cup P_{\ell}\left(\subset R_{1}\right)$, then $S_{1}$ is a Riemann subsurface satisfying Shiga's condition. Let $\hat{S}_{1}$ be a Nielsen extension of $S_{1}$ and consider the Teichmüller space $T\left(\hat{S_{1}}\right)$ and the Fenchel-Nielsen coordinate $\left(\ell_{\hat{S_{1}}}(\alpha), \theta_{\hat{S_{1}}}(\alpha)\right)$ for the pants decomposition $S_{1}=P \cup P_{n} \cup P_{l}$. Now put $S_{2}:=g\left(S_{1}\right)\left(\subset R_{2}\right)$ and let $\hat{S}_{2}$ be a Nielsen extension of $S_{2}$. Since

$$
\left|\log \frac{\ell_{\hat{S_{1}}}(\alpha)}{\ell_{\hat{S_{2}}}([g(\alpha)])}\right|<2 r
$$

holds for any $\alpha \in \mathcal{C}\left(\hat{S_{1}}\right)$,

$$
\left|\theta_{\hat{S_{1}}}\left(\alpha_{1}\right)-\theta_{\hat{S_{2}}}\left(\left[g\left(\alpha_{1}\right)\right]\right)\right|<E \cdot 2 r
$$


holds for the twist parameters $\theta_{\hat{S}_{1}}\left(\alpha_{1}\right)$ and $\theta_{\hat{S_{2}}}\left(\left[g\left(\alpha_{1}\right)\right]\right)$, where $E=E(r, M)$ is a constant depending only on $r$ and $M$ (cf. Proposition 3.3 of [2] or Lemma 4.1 of [14]). Hence, for some constant $D=D(r, M)$, the statement is true.

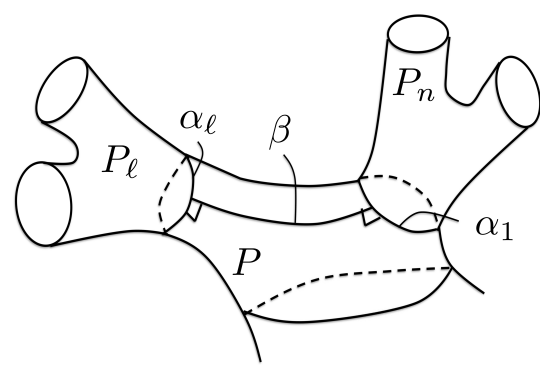

Figure 8. $S_{1}=P \cup P_{n} \cup P_{\ell}$

Similarly, the angles of the triangles in $P_{n}$ are bounded below by some constant $\theta(r, M)>0$ since the lengths of sides of triangles are bounded above by some constant depending on $r$ and $M$. Now triangulate all pairs of pants in $\mathcal{P}_{1}$ in the above way. For each triangle in each pair of pants, we define the quasiconformal mapping given by Lemma 3.1. Then we obtain a quasiconformal map $\varphi: \mathcal{P}_{1} \rightarrow \mathcal{P}_{2}$. Put $\varepsilon:=\max \{\log A, \log B, \log D\}$. Then we obtain a quasiconformal map $\varphi: R_{1} \rightarrow$ $R_{2}$ such that $K(\varphi)=1+\varepsilon \cdot F$, where $F$ is a constant depending $r, M$ and $\varepsilon$. Lastly, we show the following.

Claim 3.4. $g$ is homotopic to $\varphi$.

Proof. It is sufficient to show that for any simple closed curve $c$ in $R_{1}, g(c)$ and $\varphi(c)$ are homotopic (cf. [5], Lemma 4). Let $X$ be a finite type subsurface of $R_{1}$ that is closed (as a subset), contains $c$, and can be written as a union of triangles appearing in the given triangulation of $R_{1}$. By construction and the fact that $X$ has finite topology, there is a homotopy $H: R_{1} \times I \rightarrow R_{2}$ such that $H(x, 0)=g(x)$ and $\left.H\right|_{T \times\{1\}}=\left.\varphi\right|_{T}$ for every triangle $T$ in $X$. In particular, we see that $\varphi^{-1} \circ H$ : $R_{1} \times I \rightarrow R_{1}$ is a homotopy between $\varphi^{-1} \circ g$ and a map restricting to the identity on each triangle in $X$. In particular, for every simple closed curve $d \subset X$, we have $\varphi^{-1} \circ g(d)$ is homotopic to $d$. (See the Alexander Method of [7].) Thus $g(c)$ and $\varphi(c)$ are homotopic.

Thus $d_{T}\left(p, p^{\prime}\right)<C d_{L}\left(p, p^{\prime}\right)$ for some constant $C=C(r, M)$.

\section{REFERENCES}

[1] Daniele Alessandrini, Lixin Liu, Athanase Papadopoulos, Weixu Su, and Zongliang Sun, On Fenchel-Nielsen coordinates on Teichmüller spaces of surfaces of infinite type, Ann. Acad. Sci. Fenn. Math. 36 (2011), no. 2, 621-659. MR2865518

[2] D. Alessandrini, L. Liu, A. Papadopoulos, and W. Su, On local comparison between various metrics on Teichmüller spaces, Geom. Dedicata 157 (2012), 91-110. MR2893480

[3] Christopher J. Bishop, Quasiconformal mappings of $Y$-pieces, Rev. Mat. Iberoamericana 18 (2002), no. 3, 627-652. MR 1954866

[4] Peter Buser, Geometry and spectra of compact Riemann surfaces, Progress in Mathematics, vol. 106, Birkhäuser Boston, Inc., Boston, MA, 1992. MR.1183224 
[5] Adam Lawrence Epstein, Effectiveness of Teichmüller modular groups, In the tradition of Ahlfors and Bers (Stony Brook, NY, 1998), Contemp. Math., vol. 256, Amer. Math. Soc., Providence, RI, 2000, pp. 69-74. MR1759670

[6] Ozgur Evren, The Length Spectrum Metric on the Teichmuller Space of a Flute Surface, ProQuest LLC, Ann Arbor, MI, 2013. Thesis (Ph.D.)-City University of New York. MR3152661

[7] Benson Farb and Dan Margalit, A primer on mapping class groups, Princeton Mathematical Series, vol. 49, Princeton University Press, Princeton, NJ, 2012. MR2850125

[8] Erina Kinjo, On Teichmüller metric and the length spectrums of topologically infinite Riemann surfaces, Kodai Math. J. 34 (2011), no. 2, 179-190. MR2811639

[9] Erina Kinjo, On the length spectrum metric in infinite dimensional Teichmüller spaces, Ann. Acad. Sci. Fenn. Math. 39 (2014), no. 1, 349-360. MR.3186819

[10] Zhong Li, Teichmüller metric and length spectrums of Riemann surfaces, Sci. Sinica Ser. A 29 (1986), no. 3, 265-274. MR855233

[11] Liu Lixin, On the length spectrum of non-compact Riemann surfaces, Ann. Acad. Sci. Fenn. Math. 24 (1999), no. 1, 11-22. MR1678001

[12] Lixin Liu and Athanase Papadopoulos, Some metrics on Teichmüller spaces of surfaces of infinite type, Trans. Amer. Math. Soc. 363 (2011), no. 8, 4109-4134. MR2792982

[13] Lixin Liu, Zongliang Sun, and Hanbai Wei, Topological equivalence of metrics in Teichmüller space, Ann. Acad. Sci. Fenn. Math. 33 (2008), no. 1, 159-170. MR2386845

[14] Hiroshige Shiga, On a distance defined by the length spectrum of Teichmüller space, Ann. Acad. Sci. Fenn. Math. 28 (2003), no. 2, 315-326. MR.1996441

[15] Tuomas Sorvali, The boundary mapping induced by an isomorphism of covering groups, Ann. Acad. Sci. Fenn. Ser. A I 526 (1972), 31. MR0328066

[16] W. P. Thurston, Minimal stretch maps between hyperbolic surfaces; 1986 preprint converted to 1998 eprint, http://arxiv.org/pdf/math/9801039

Department of Mathematics, Tokyo Institute of Technology, Oh-OKayama 2-12-1, MEGURO-KU, TOKYO 152-8551, JAPAN

Email address: kinjo.e.aa@m.titech.ac.jp 